\title{
GEOMETRY OF THE LOCUS OF POLYNOMIALS OF DEGREE 4 WITH ITERATIVE ROOTS
}

\author{
BEATA STRYCHARZ-SZEMBERG, TOMASZ SZEMBERG
}

\begin{abstract}
We study polynomial iterative roots of polynomials and describe the locus of complex polynomials of degree 4 admitting a polynomial iterative square root.
\end{abstract}

\section{INTRODUCTION}

We begin by recalling some basic notions about iterations. Let $M$ be an arbitrary set and let $f: M \longrightarrow M$ be a function.

Definition 1.1. The iterates of $f$ are defined recursively by

- $f^{0}(x)=x$ for all $x \in M$;

- $f^{n+1}(x)=f\left(f^{n}(x)\right)$ for all $x \in M$ and $n \geq 0$.

Example 1.2. Let $f: \mathbb{C} \longrightarrow \mathbb{C}$ be a linear polynomial $f(z)=a z+b$. Then

$$
\begin{aligned}
& f^{0}(z)=z \\
& f^{1}(z)=a z+b \\
& f^{2}(z)=a(a z+b)+b=a^{2} z+a b+b \\
& f^{3}(z)=a^{3} z+a^{2} b+a b+b \\
& \vdots \\
& f^{n}(z)=a^{n} z+\left(a^{n-1}+a^{n-2}+\cdots+a+1\right) b .
\end{aligned}
$$

It is natural to ask if a given function $g$ can be represented as an iterate of another function $f$. If this is the case, then we say that $f$ is an iterative root of $g$. This is defined precisely below.

Definition 1.3. We say that a function $f$ is an iterative root of order $r \geq 2$ of a function $g$, if the following functional equation is satisfied

$$
f^{r}=g
$$

and $r$ is the least integer for which this equation holds.

The problem of the existence of iterative roots appear in the literature already at the beginning of the 19th century, notably in the works of Abel and Babbage, see eg. Bab. In the present note we consider this problem for complex polynomials in one variable. Iterations of polynomials of more variables were studied recently in [CSZ].

\section{Complex polynomials and Algebraic Sets}

We recall some notions necessary in the further considerations.

Definition 2.1. a) A complex polynomial $g(z)$ of one variable $z$ of degree $d$ is an expression of the form

$$
g(z)=b_{d} z^{d}+b_{d-1} z^{d-1}+\cdots+b_{1} z+b_{0},
$$

with $b_{0}, \ldots, b_{d} \in \mathbb{C}$ and $b_{d} \neq 0$. If $d=0$, then also $b_{0}=0$ is allowed. 
b) The set $P(d)$ of polynomials of degree $\leq d$ is a vector space of dimension $d+1$ with natural coordinates $\left(b_{d}, b_{d-1}, \ldots, b_{0}\right)$.

c) The set of normalized polynomials $P_{n}(d)$ of degree $d$ (i.e. those with $b_{d}=1$ ) is an affine subspace of $P(d)$, which we identify with $\mathbb{C}^{d}$ via natural coordinates $\left(b_{d-1}, b_{d-2}, \ldots, b_{0}\right)$.

Complex polynomials in more variables are defined similarly. Their set has a structure of ring and is denoted usually by $\mathbb{C}\left[z_{1}, \ldots, z_{n}\right]$.

Definition 2.2. An (affine) algebraic set in $\mathbb{C}^{n}$ is a set $X$ defined by a (finite) number of polynomial equations

$$
X=\left\{\begin{array}{ccc}
f_{1}\left(z_{1}, \ldots, z_{n}\right) & = & 0 \\
f_{2}\left(z_{1}, \ldots, z_{n}\right) & = & 0 \\
\vdots & \vdots & \vdots \\
f_{k}\left(z_{1}, \ldots, z_{n}\right) & = & 0
\end{array}\right.
$$

where $f_{1}, \ldots, f_{k} \in \mathbb{C}\left[z_{1}, \ldots, z_{n}\right]$.

One of fundamental theorems in algebraic geometry is Hilbert's Nullstellensatz [Hul, Theorem 1.16]. It associates to any algebraic subset $X \subset \mathbb{C}^{n}$ its vanishing ideal $I(X)$. The transcendence degree of the quotient field $\mathbb{C}(X)$ of the quotient ring $\mathbb{C}\left[z_{1}, \ldots, z_{n}\right] / I(X)$ is the dimension of $X$.

\section{ITERATIVE ROOTS OF COMPLEX POLYNOMIALS}

Motivated by the ideas recalled in the Introduction we are led to the following problem.

Question 3.1. Do complex polynomials in one variable of given degree d admit iterative roots?

This question is very general and a little bit ambiguous at the same time. First of all note, that if $f$ is a polynomial of degree $e$, then $f^{r}$ is a polynomial of degree $e^{r}$, so there are obvious constrains involving the degree for the existence of polynomial iterative roots. For example a polynomial whose degree $d$ is a prime cannot admit any polynomial roots with $r \geq 2$.

Sending a polynomial $f \in P(d)$ to its $r$-th iterate $f^{r} \in P\left(d^{r}\right)$ is an algebraic mapping. This means the coefficients of $f^{r}$ are polynomial expressions in coefficients of $f$. The image is thus an algebraic set and its dimension is bounded by $d+1$, the dimension of $P(d)$, whereas the dimension of $P\left(d^{r}\right)$ is $\left(d^{r}+1\right)$. This implies that a general polynomial of degree $d^{r}$ has no polynomial root of order $r$. It is interesting to have some criteria. This motivated our Theorem 4.2 .

On the other hand, one might wonder if given a polynomial $g(z)$ there exists arbitrary function such that

$$
f^{r}=g
$$

with no assumptions whatsoever on the regularity of $f$. This question seems to be much harder. We collect together what is known about Question 3.1 to put our result in a perspective.

3.1. Constant polynomials. A polynomial $g$ of degree 0 is a constant function $g(z)=b_{0}$. It is clear that $g$ is its own iterative root of arbitrary degree i.e. $g^{r}=g$ for all $r \geq 1$. 
3.2. Linear polynomials. A linear polynomial $g: \mathbb{C} \ni z \longrightarrow a z+b \in \mathbb{C}$ with $a \neq 0$ has iterative roots of arbitrary order $r$.

Proof. If $a \neq 1$, then set simply $c:=a^{\frac{1}{r}}$ (we take one of the roots of order $r$ of $a$ ), $d:=\frac{b}{1+c+\cdots+c^{r-1}}=b \frac{1-c}{1-a}$ and define $f(z)=c z+d$. Then $f$ is an iterative root of $g$ of order $r$, compare Example 1.2 .

If $a=1$ i.e. $g(z)=z+b$, then clearly $f(z)=z+\frac{b}{r}$ is the iterative of $g$ of order $r$.

If $b=0$, then for $\alpha$ an arbitrary root of 1 of order $r$ the polynomial $f(z)=\alpha \cdot z$ is an iterative root of $g(z)=z$.

It follows from the proof that a complex linear polynomial admits at least $r$ distinct iterative roots of order $r$, which are linear polynomials as well. One cannot however hope for an iterative version of the fundamental theorem of algebra. Indeed, the identity $g(z)=z$ has infinitely many polynomial iterative square roots of the form $f(z)=-z+b$ with arbitrary $b \in \mathbb{C}$.

3.3. Quadratic polynomials. As the degree $d=2$ is a prime, there cannot exist any polynomial iterative roots. On the other hand in [RSS] Rice, Schweizer and Sklar proved a surprising result to the effect that a quadratic complex polynomial does not admit any iterative root i.e., with no assumption on the regularity of a root.

Theorem 3.2 (Rice-Schweizer-Sklar). Let $g(z)$ be a quadratic complex polynomial and $r \geq 2$ an integer. Then there does not exist any function $f: \mathbb{C} \longrightarrow \mathbb{C}$ such that $f^{r}=g$.

Remark 3.3. Note that the result strongly depends on the ground field. For example there exist iterative square roots of some real quadratic polynomials, see [Bro] for details.

3.4. Cubic polynomials. The degree is again a prime, so there are no polynomial roots. Choczewski and Kuczma claim in [C-K] that the degree 3 counterpart of Theorem 3.2 holds. However the factorization in [C-K, Formula 20] is false and the rest of their argument fails, so that we don't know for sure.

3.5. Quartic polynomials. Obviously there are quartic polynomials which are iterative squares of quadratic polynomials. As remarked already above a general quartic polynomial is not an iterative square, so it is natural to ask how to distinguish those which are among all the others. In this note we show that there exists a rational surface $S$ in the space $P_{n}(4)$ of normalized polynomials of degree 4 , which parametrizes iterative squares. The precise statement is given in Theorem 4.2, which is our main result. Degree 4 is in a sense the first interesting case because there are quartic polynomials which have polynomial iterative roots of order 2 and such which haven't.

3.6. An overview. We summarize facts presented in this section in the following table.

\begin{tabular}{|c|c|c|}
\hline degree & which have polynomial iterative roots & which have iterative roots \\
\hline 0 & all & all \\
1 & all & all \\
2 & none & none \\
3 & none & none $(?)$ \\
4 & a proper algebraic subset & $? ? ?$ \\
\hline
\end{tabular}


The following problem is quite natural (the evidence might be to sparse to rise a conjecture). We stress again, that in view of Remark 3.3 it is crucial, that we are over the complex numbers.

Question 3.4. Let $g$ be a complex polynomial in one variable which has an iterative root of order $r$. Does there exist a polynomial iterative root of $g$ of order $r$ ?

Note that one cannot hope that any iterative root of a polynomial is a polynomial itself. For example the identity $g(z)=z$ has also rational functions $f(z)=\frac{a}{z}$ with arbitrary $a \in \mathbb{C}, a \neq 0$ as iterative square roots (and more complicated functions as well).

\section{THE MAIN RESUlT}

We begin by showing that it is enough to consider normalized polynomials i.e. to work in the space $P_{n}(4)$. Let $L(z)=a z+b$ be a linear polynomial, $a \neq 0$. Then $L$ is a bijective mapping of the complex plane with the inverse $L^{-1}(z)=\frac{1}{a} z-\frac{b}{a}$. We have the following observation.

\section{Lemma 4.1. Let}

$$
g(z)=b_{d} z^{d}+b_{d-1} z^{d-1}+\cdots+b_{1} z+b_{0}
$$

be a complex polynomial of degree $d$. There exists a normalized polynomial $g_{n}$, which is linear conjugate to $g$ i.e. $g_{n}=L^{-1} \circ g \circ L$ for some $L(z)=a z+b$ as above.

Proof. Elementary calculation shows that

$$
\left(L^{-1} \circ g \circ L\right)(z)=\left(b_{d} \cdot a^{d-1}\right) z^{d}+\text { terms of lower degree. }
$$

Since we work over the complex numbers there exists $a \in \mathbb{C}$ such that

$$
b_{d} \cdot a^{d-1}=1
$$

and the assertion of the Lemma follows.

It is a general fact, that existence of iterative roots is invariant under conjugation. Indeed, assume that

$$
\gamma=\varphi^{r}
$$

holds and let $\lambda$ be any bijection. Then $\lambda^{-1} \circ \gamma \circ \lambda$ has $\lambda \circ \varphi \circ \lambda$ as its iterative root of order $r$. Hence it is enough to consider normalized polynomials.

Let $f(z)=z^{2}+a_{1} z+a_{0}$ be a normalized quadratic polynomial. Then

$$
f^{2}(z)=z^{4}+2 a_{1} \cdot z^{3}+\left(2 a_{0}+a_{1}^{2}+a_{1}\right) \cdot z^{2}+\left(2 a_{1} a_{0}+a_{1}^{2}\right) \cdot z+\left(a_{0}^{2}+a_{1} a_{0}+a_{0}\right) .
$$

This calculation can be interpreted in terms of the polynomial mapping

$$
\varphi: P_{n}(2)=\mathbb{C}^{2} \ni\left(a_{1}, a_{0}\right) \rightarrow\left\{\begin{array}{rl}
b_{3} & =2 a_{1} \\
b_{2} & =2 a_{0}+a_{1}^{2}+a_{1} \\
b_{1} & =2 a_{1} a_{0}+a_{1}^{2} \\
b_{0} & =a_{0}^{2}+a_{1} a_{0}+a_{0}
\end{array} \in \mathbb{C}^{4}=P_{n}(4) .\right.
$$

We denote the image of this mapping by $S$.

Eliminating the variables $a_{0}$ and $a_{1}$ in the above equations either by hand or using Singular [DGPS] as we did, leads to the following equations for $S$ :

$$
\begin{aligned}
b_{3}^{4}-8 b_{2} b_{3}^{2}-12 b_{3}^{2}+16 b_{2}^{2}+32 b_{2}-16 b_{3}-64 b_{0} & =0 ; \\
b_{3}^{3}-4 b_{2} b_{3}+8 b_{1} & =0
\end{aligned}
$$

Up to rearranging the terms we just proved our main result. 
Theorem 4.2. Let $g(z)=z^{4}+b_{3} z^{3}+b_{2} z^{2}+b_{1} z+b_{0}$ be a normalized complex quartic polynomial. There exists a normalized polynomial $f(z)=z^{2}+a_{1} z+a_{0}$ of degree 2 such that $f^{2}=g$ if and only if

$$
b_{0}=\frac{1}{64}\left(b_{3}^{4}-8 b_{2} b_{3}^{2}-12 b_{3}^{2}+16 b_{2}^{2}+32 b_{2}-16 b_{3}\right) \quad \text { and } \quad b_{1}=-\frac{1}{8}\left(b_{3}^{3}-4 b_{2} b_{3}\right) .
$$

In this case

$$
a_{1}=\frac{1}{2} b_{3} \text { and } a_{0}=-\frac{1}{8} b_{3}^{2}-\frac{1}{4} b_{3}+\frac{1}{2} b_{2}
$$

Remark 4.3. Whereas equations (2) distinguish iterative squares exactly in the same manner as for example the vanishing of the discriminant of a polynomial distinguishes polynomials with multiple roots, the forms (3) show that a polynomial square root can be easily computed effectively.

Remark 4.4. As pointed out by the referee it might happen that a normalized polynomial has an iterative root which is not normalized. Indeed, if $\alpha$ is an arbitrary cubic root of 1 , then the second iterate of $h(z)=\alpha z^{2}+\beta_{1} z+\beta_{0}$ is a normalized polynomial of degree 4 .

As we saw in Lemma 4.1, the polynomials $h(z)$ in Remark 4.4 and $f(z)$ in Theorem 4.2 are linearly conjugate, for example by $L_{\alpha}(z)=\alpha \cdot z$ which sends

$$
f(z)=z^{2}+a_{1} z+a_{0} \text { to } h(z)=\alpha z^{2}+a_{1} z+\alpha^{2} a_{0},
$$

i.e. $\beta_{1}=a_{1}$ and $\beta_{0}=\alpha^{2} a_{0}$. The second iterates of the above polynomials are normalized polynomials, of course again conjugate by $L_{\alpha}$.

From now on, we assume that $\alpha$ is a primitive cubic root. Starting with $h(z)$ instead of $f(z)$ we get a mapping $\varphi_{\alpha}$ similarly as in (1)

$$
\varphi_{\alpha}: P_{n}(2)=\mathbb{C}^{2} \ni\left(a_{1}, a_{0}\right) \rightarrow\left\{\begin{array}{rl}
b_{3} & =2 \alpha^{2} a_{1} \\
b_{2} & =2 \alpha^{2} a_{0}+\alpha a_{1}^{2}+\alpha a_{1} \\
b_{1} & =2 \alpha a_{1} a_{0}+a_{1}^{2} \\
b_{0} & =\alpha a_{0}^{2}+a_{1} a_{0}+a_{0}
\end{array} \in \mathbb{C}^{4}=P_{n}(4) .\right.
$$

and by the same token we have the map $\varphi_{\alpha^{2}}$. We denote the images of these maps by $S_{\alpha}$ and $S_{\alpha^{2}}$ respectively. It is clear that the conjugation by $L_{\alpha}$, respectively by $L_{\alpha^{2}}(z)=\alpha^{2} \cdot z$ induces isomorphisms of $S$ with $S_{\alpha}$ and $S_{\alpha^{2}}$. It suffices to understand the geometry of $S$. It is surprisingly simply. Since $S$ is the graph of a polynomial mapping in variables $b_{2}$ and $b_{3}$, it is a rational surface. It has an isolated singularity in the point $(0,0,0,0)$ which corresponds to the polynomial $z^{4}$. This polynomial sits in the intersection $C:=S \cap S_{\alpha} \cap S_{\alpha^{2}}$, hence it has three distinct polynomial square roots: $z^{2}, \alpha \cdot z^{2}$ and $\alpha^{2} \cdot z^{2}$. It is natural to ask if there are more such polynomials.

\section{Multiple Roots And GeOmetry}

Here we show that $C$ is an irreducible rational curve, hence in particular there is a one parameter family of quartic polynomials with triple iterative roots of order 2 .

Proposition 5.1. Let $g(z)=z^{4}+\beta \cdot z^{3}+\frac{3}{8} \beta^{2} \cdot z^{2}+\frac{1}{16} \beta^{3} \cdot z+\frac{1}{256} \beta^{4}-\frac{1}{4} \beta$ with $\beta \in \mathbb{C}$ arbitrary. Then $g$ has three distinct iterative roots of order 2 .

Proof. A naive idea to describe all polynomials with multiple iterative roots would be to consider pairwise intersections of surfaces $S, S_{\alpha}$ and $S_{\alpha^{2}}$. This is doable but the computations are nasty. Here we compute instead iterates of quadratic polynomials. Let

$$
f_{\alpha}(z)=\alpha z^{2}+b_{1} z+b_{0},
$$


then we have

(4) $f^{2}(z)=\alpha^{3} z^{4}+2 \alpha^{2} b_{1} z^{3}+\left(2 \alpha^{2} b_{0}+\alpha b_{1}^{2}+\alpha b_{1}\right) z^{2}+\left(2 \alpha b_{0} b_{1}+b_{1}^{2}\right) z+\left(\alpha b_{0}^{2}+b_{0} b_{1}+b_{0}\right)$.

It is clear that $f^{2}(z)$ is a normalized polynomial if and only if $\alpha$ is a cubic root of 1 . We assume that $\alpha$ is a primitive root. We write

$$
f_{1}(z)=z^{2}+a_{1} z+a_{0}, \text { and } f_{\alpha^{2}}(z)=\alpha^{2} z^{2}+c_{1} z+c_{0} .
$$

We get formula for $f_{1}^{2}(z)$, respectively $f_{\alpha^{2}}^{2}(z)$ replacing in (4) $\alpha$ by 1 , respectively by $\alpha^{2}$.

With second iterates of $f_{1}, f_{\alpha}$ and $f_{\alpha^{2}}$ computed, we compare coefficients at corresponding powers of $z$. We begin with $f_{1}$ and $f_{\alpha}$, which corresponds to computing the intersection of $S$ with $S_{\alpha}$. We get the following system of equations

$$
\left\{\begin{aligned}
a_{1} & =\alpha^{2} b_{1} \\
2 a_{0}+a_{1}^{2}+a_{1} & =2 \alpha^{2} b_{0}+\alpha b_{1}^{2}+\alpha b_{1} \\
2 a_{1} a_{0}+a_{1}^{2} & =2 \alpha b_{0} b_{1}+b_{1}^{2} \\
a_{0}^{2}+a_{1} a_{0}+a_{0} & =\alpha b_{0}^{2}+b_{0} b_{1}+b_{0}
\end{aligned}\right.
$$

Using elimination (we were again aided by Singular but it can be done also directly) we get

$$
\left\{\begin{aligned}
b_{1} & =\alpha a_{1} \\
b_{0} & =\frac{1}{4} \alpha\left(a_{1}-2 \alpha^{2}\right) a_{1} \\
a_{0} & =\frac{1}{4}\left(a_{1}-2\right) a_{1}
\end{aligned}\right.
$$

With $\beta:=2 a_{1}$ we can write two iterative square roots of $g(z)$ explicitly as

$$
\begin{aligned}
& f_{1}(z)=z^{2}+\frac{1}{2} \beta z-\frac{1}{16} \beta\left(\beta \alpha^{2}+\beta \alpha+4\right), \\
& f_{\alpha}(z)=\alpha z^{2}+\frac{1}{2} \beta \alpha z-\frac{1}{16} \beta\left(\beta+\beta \alpha^{2}+4\right) .
\end{aligned}
$$

The intersection curve $\Gamma:=S \cap S_{\alpha}$ is thus parametrized by the polynomial mapping

$$
\mathbb{C} \ni \beta \rightarrow\left(\beta, \frac{3}{8} \beta^{2}, \frac{1}{16} \beta^{3}, \frac{1}{256} \beta^{4}-\frac{1}{4} \beta\right) \in P_{n}(4)=\mathbb{C}^{4} .
$$

In particular this curve is rational and irreducible. It is not hard to check in the same way, that $S_{\alpha^{2}}$ intersects $S$ and $S_{\alpha}$ exactly along $\Gamma$. Alternatively one could argue with the cyclic group of order 3 generated by $\alpha$ acting on the whole picture. In any case $\Gamma=C$ and the third iterative root of $g$ is

$$
f_{\alpha^{2}}(z)=\alpha^{2} z^{2}+\frac{1}{2} \beta \alpha^{2} z-\frac{1}{16} \beta(\beta+\beta \alpha+4) .
$$

Proposition 5.1 has the following somewhat surprising consequence.

Corollary 5.2. A normalized quartic polynomial has

a) either no polynomial iterative square root (it lies in the complement of the set $S \cup$ $\left.S_{\alpha} \cup S_{\alpha^{2}} \subset P_{n}(4)\right)$;

b) or one polynomial square root (it is a point in $\left.\left(S \cup S_{\alpha} \cup S_{\alpha^{2}}\right) \backslash C\right)$ );

c) or three polynomial square roots (it is a point on the curve $C$ ).

It would be interesting to know if the geometric picture carries over to the general setting i.e. if the geometry of the set of polynomials of degree $d^{r}$, which are $r$-th iterative powers of polynomials of degree $d$ has a similar description and if one can characterize polynomials with multiple iterative polynomial roots. We hope to come back to this question in the next future. 
Acknowledgements. We would like to thank the anonymous referees for helpful remarks which greatly improved the exposition of the paper. The second author was partially supported by a MNiSW grant N N201 388834 .

\section{REFERENCES}

[Bab] Babbage, C.: Examples of the solution of functional equations. Cambridge 1820

[Bro] Bronshtein, E. M.: On an iterative square root of a quadratic trinomial. (Russian) Geometric problems in the theory of functions and sets (Russian), 24-27, Kalinin. Gos. Univ., Kalinin, 1989.

[CSZ] Chen, X., Shi, Y., Zhang, W.: Planar Quadratic Degree-Preserving Maps and Their Iteration, Result. Math. 55 (2009), 39-63

[C-K] Choczewski, B.; Kuczma, M.: On iterative roots of polynomials. European conference of iteration theory - Lisboa'91 (1992), 59-67

[DGPS] Decker, W.; Greuel, G.-M.; Pfister, G.; Schönemann, H.: Singular 3-1-1 — A computer algebra system for polynomial computations. http://www.singular.uni-kl.de (2010).

[Hul] Hulek, K.: Elementary algebraic geometry. Student Mathematical Library, 20. AMS, Providence, RI, 2003

[RSS] Rice, R. E.; Schweizer, B.; Sklar, A.: When is $f(f(z))=a z^{2}+b z+c$ ? Amer. Math. Monthly 87 (1980), 252-263.

[S-S] Schweizer, B.; Sklar, A.: Invariants and equivalence classes of polynomials under linear conjugacy. Contributions to general algebra, 6, 253-257, Hölder-Pichler-Tempsky, Vienna, 1988.

Beata Strycharz-Szemberg, Instytut Matematyki, Politechnika Krakowska, Warszawska 24, PL-31-155 Kraków, Poland

E-mail address: szemberg@pk.edu.pl

Tomasz Szemberg, Instytut Matematyki UP, Podchorażych 2, PL-30-084 Kraków, Poland

Current address: Albert-Ludwigs-Universität Freiburg, Mathematisches Institut, Eckerstraße 1, D-79104 Freiburg, Germany

E-mail address: tomasz.szemberg@uni-due.de 